BULL. AUSTRAL. MATH. SOC.

$47 \mathrm{HI}, 34 \mathrm{HO}, 47 \mathrm{~B} 44,47005$

VOL. $10(1974), 31-37$.

\title{
Nonlinear differential equations in reflexive Banach spaces
}

\section{W.E. Fitzgibbon}

Let $X$ be a reflexive Banach space and $\{A(t) \mid t \in[0, T]\}$ be a family of weakly continuous operators which map $X$ to $X$. Conditions are provided which guarantee the existence and the uniqueness to the Cauchy initial value problem $u^{\prime}(t)+A(t) u(t)=0 ; u(0)=x$.

\section{Introduction}

In this paper we shall be concerned with the existence of solutions to the Cauchy initial value problem,

$$
u^{\prime}(t)+A(t) u(t)=0 ; u(0)=x,
$$

where $\{A(t) \mid t \in[0, T]\}$ is a family of operators which map a reflexive Banach space $X$ to itself. Basically we require that the operator $A(\cdot):[0, T] \times X \rightarrow X$ be weakly continuous and that for each $t \in[0, T]$ the operator $A(t)$ satisfy a modified accretivity condition. In [1] Browder provides a local solution to (1.1) in case $X$ is a complex Hilbert space. More recently, Diaz and Weinacht [3] and Medeiros [11] discuss the uniqueness of solutions to (1.1) in Hilbert spaces; Goldstein in [6] extends their results to general Banach spaces; and Chow and Schuur [2] guarantee local existence to (1.1) in case $X$ is a separable reflexive, Banach space. In [5] the author establishes the global existence of solutions to $(1.1)$ in case $A(t) \equiv A$ is accretive. 


\section{Preliminaries}

Throughout this paper $X$ will denote a Banach space and $\|\cdot\|$ will be its norm. The dual space of $X$ will be $X^{*}$.

DEFINITION 2.1. Let $X$ be a Banach space, then the duality map $F: X \rightarrow 2^{X^{*}}$ is defined in the following manner: if $x \in X$, then $x^{*} \in F(x)$ iff $x^{*}(x)=\|x\|^{2}$ and $\left\|x^{*}\right\|=\|x\|$.

In general the duality map is not single valued; however, in [7], Kato shows that if $X$ is a Banach space having uniformly convex dual $X^{*}$, then the duality map is uniformly continuous on bounded subsets of $X$.

The following definition makes clear our notions of operator continuity.

DEFINITION 2.2. Let $\{A(t) \mid t \in[0, T]\}$ be a family of operators which map $X$ to $X$. Then $\{A(t) \mid t \in[0, T]\}$ is said to be weak $z y$ continuous provided that $t_{n}+t_{0}$ and $x_{n}-x_{0}$ imply $A\left(t_{n}\right) x_{n} \rightarrow A\left(t_{0}\right) x_{0}$. If $t_{n}+t_{0}$ and $x_{n} \rightarrow x_{0}$ implies $A\left(t_{n}\right) x_{n} \rightarrow A\left(t_{0}\right) x_{0}$, then $\{A(t) \mid t \in[0, T]\}$ is said to be demi-continuous.

We now define an accretive operator and give two useful characterizations of accretive operators.

DEFINITION 2.3. Let $X$ be a Banach space and $A$ an operator mapping a subset of $X$ to $X$; then $A$ is said to be accretive provided (2.4), $\|x+\lambda A x-(y+\lambda A y)\| \geq\|x-y\|$ whenever $x, y \in D(A)$ and $\lambda \geq 0$.

Although Definition 2.3 is easily stated it is difficult to apply. In [8] Kato shows that an operator is accretive if $\langle A x-A y, f\rangle \geq 0$ for $x, y \in D(A)$ and some $f \in F(x-y)$ where $F$ is the duality map. An accretive operator $A$ is said to be strongly accretive provided that $\langle A x-A y, f\rangle \geq 0$ for all $f \in F(x-y)$. It is easily shown that weakly continuous accretive operators are strongly accretive. Martin [10] shows that if $A$ is strongly accretive, then

$$
\lim _{h \rightarrow 0^{+}}(\|x-y-h(A x-A y)\|-\|x-y\|) / h \leq 0 \text { for all } x, y \in D(A) \text {. }
$$

We now make precise our notion of strong solutions to the Cauchy problem. 
DEFINITION 2.5. A function $u(\cdot):[0, T] \rightarrow X$ is said to be a strong solution to the Cauchy problem

$$
u^{\prime}(t)+A(t) u(t)=0 ; u(0)=x,
$$

provided that $u$ is Lipschitz continuous on $[0, T], u(0)=x, u$. is strongly differentiable almost everywhere and $u^{\prime}(t)+A(t) u(t)=0$ for $t \in[0, T]$ almost everywhere.

LEMMA 2.7. Let $X$ be a Banach space and $g$ be a function from the number interval $(a, b)$ to $X$. Define $p(t)=\|g(t)\|$ for $t \in[a, b]$; then if $g^{\prime^{+}}(t)$ exists, $p^{{ }^{+}}(t)$ exists and

$$
p^{{ }^{+}}(t)=\lim _{h \rightarrow 0^{+}}\left\|g(t)+h g^{{ }^{+}}(t)\right\|-\|g(t)\| / h \text {. }
$$

\section{Existence of solutions}

The following lemma provides a local solution to Definition 2.5 .

LEMMA 3.1. Let $X$ be a reflexive Banach space and suppose that $\{A(t) \mid t \in[0, T]\}$ is a weakly continuous family of operators which mop $X$ to $X$; then there is a finite interval $\left[0, T_{0}\right]$ such that the Cauchy problem has a strong solution on $\left[0, T_{0}\right]$.

Proof. Let $x \in X$. By virtue of the weak continuity of $\{A(t) \mid t \in[0, T]\}$ there exist $T_{1}, R$ and $K_{1}>0$ such that if $0 \leq t \leq T_{1}$ and $y \in S_{R}(x)$ then $\|A(t) y\| \leq K$. Choose $T_{0}=\min \left\{R / K, T_{1}\right\}$. Let $\varepsilon_{n}+0$. We shall recursively define a sequence of functions which solve the approximate equations

$$
\begin{aligned}
u_{n}^{\prime}(t)+A(t) u_{n}\left(t-\varepsilon_{n}\right)=0 ; u(0)=x ; & u_{n}(t)=\left\{\begin{array}{r}
x \text { if } t<0, \\
x-\int_{0}^{t} A(s) u_{n}\left(8-\varepsilon_{n}\right) d s \text { if } t \in\left[j \varepsilon_{n},(j+1) \varepsilon_{n}\right], \\
j=0, \ldots,\left[T_{0} / \varepsilon_{n}\right]-1 .
\end{array}\right.
\end{aligned}
$$

We argue that $u_{n}(t) \in S_{R}(x)$. If $t \in\left[0, \varepsilon_{n}\right]$ then $\left\|u_{n}(t)-x\right\| \leq t \sup _{s \in[0, T]}\|A(s) x\| \leq(R / K) K=R$. If we assume the desired 
result for $t \in\left[0, j \varepsilon_{n}\right]$ and consider $t \in\left[0,(j+1) \varepsilon_{n}\right]$, we have $\left\|u_{n}(t)-x\right\| \leq\left\|\int_{0}^{t} A(s) u_{n}\left(s-\varepsilon_{n}\right) d s\right\| \leq \operatorname{tmax}\left\{\left\|A(s) u_{n}\left(s-\varepsilon_{n}\right)\right\| \mid s \in\left[0,(j+1) \varepsilon_{n}\right]\right\}$. By observing that $\left\|u_{n}(t)-u_{n}(\tau)\right\| \leq \int_{\tau}^{t}\left\|A(s) u_{n}\left(s-\varepsilon_{n}\right)\right\| d s \leq|t-\tau| K$ we see that the sequence is uniformly Lipschitz continuous in $t$.

We now claim that there is a subsequence $\left\{u_{n^{\prime}}(t)\right\}$ of $\left\{u_{n}(t)\right\}$ such that $\left\{u_{n},(t)\right\}$ converges weakly to a Lipschitz continuous function $\{u(t)\}$. The argument of Lemna 2.1, [5], is directly applicable to establish this convergence.

$$
\text { Since } u_{n}\left(t-\varepsilon_{n}\right) \rightarrow u(t), A(t) u_{n}\left(t-\varepsilon_{n}\right) \rightarrow A(t) u(t) \text {. If } f \in X^{*} \text { we }
$$
take limits of the equation,

$$
\left(u_{n}(t), f\right)=(x, f)-\int_{0}^{t}\left(A(s) u_{n}\left(s-\varepsilon_{n}\right), f\right) d s
$$

to obtain

$$
(u(t), f)=(x, f)-\int_{0}^{t}(A(s) u(s), f) d s \text { for } t \in\left[0, T_{0}\right] .
$$

Applying standard techniques to (3.3) yields

$$
u(t)=x-\int_{0}^{t} A(s) u(s) d s \text { for } t \in\left[0, T_{0}\right],
$$

and hence that

$$
d u(t) / d t+A(t) u(t)=0 \text { for } t \in\left[0, T_{0}\right] \text { almost everywhere. }
$$

We now place further conditions on $\{A(t) \mid t \in[0, T]\}$ which allow us to extend the local solution of Lemme 3.1 .

THEOREM 1. Let $X$ be a reflexive Banach space and suppose that $\{A(t) \mid t \in[0, T]\}$ is a weakly continuous farmily of operators which maps $X$ to $X$. Further assume that for each $t \in[0, T]$ the operator $A(t)+(1 / t) I$ is accretive. Then there is a strong solution the cauchy initial value problem, Definition 2.5, on $[0, T]$. 
Proof. From the preceding lemma it is clear that there exists a local solution to Definition 2.5 on a maximal interval of existence $\left[0, T_{0}\right]$. We wish to argue that $T_{0}<T$ leads to a contradiction. Let $0<t_{0}<T_{0}$ and define $p(t)=\|u(t)\|$. By virtue of equation (2.4) and Lemmas 2.7 and 3.1 we have

$$
\begin{aligned}
p^{\prime+}(t)=\lim _{h \rightarrow 0^{+}}(\|u(t)-h A(t) u(t)-u(t)\|) / h & \\
& \leq \lim _{h \rightarrow 0^{+}}(\|u(t)-h(A(t) u(t)+(1 / t) u(t)-A(t) 0)\|-\|u(t)\|) / h \\
& \leq \sup _{t \in[0, T]}\|A(t) 0\|+(1 / t)\|u(t)\| \\
& \leq(1 / t)\|u(t)\|+M \text { for some } M>0 .
\end{aligned}
$$

Thus

$$
((1 / t)\|u(t)\|)^{1+} \leq(1 / t) M ;
$$

integrating on $\left(t_{0}, t\right)$ we have

$$
(1 / t)\|u(t)\| \leq\left(1 / t_{0}\right)\left\|u\left(t_{0}\right)\right\|+M^{\prime} \text { for some } M^{\prime} \text {. }
$$

Thus there is an $N>0$ such that $\|u(t)\|<N$ for $t \in\left[\dot{0}, T_{0}\right]$. Since $A(\cdot)$ maps bounded subsets of $[0, T] \times X$ to bounded subsets of $X$, there exists an $N_{1}$ such that $\int_{0}^{t}\|A(s) u(s)\| d s<N_{1}$ for $t \in\left[0, T_{0}\right)$. This implies that $\int_{0}^{t} A(s) u(s) d s$ exists for $t \in\left[0, T_{0}\right)$ and by virtue of the continuity of the integral we can define $u\left(T_{0}\right)=\lim _{t \rightarrow T_{0}} \int_{0}^{t} A(s) u(s) d s-x$. Lema 3.1 can be applied to continue the solution $u(t)$ past $T_{0}$ and thereby contradict the definition of $T_{0}$. In [6] Goldstein insures the uniqueness of the solution $u(t)$.

If we require that $X$ have uniformly convex dual and that each $A(t)$ is accretive we can relax the continuity requirement. The following theorem is an extension of a time independent result of Kato [7]. 
THEOREM 2. Let $X$ be a Banach space such that $X^{*}$ is uniformly convex and let $\{A(t) \mid t \in[0, T]\}$ be a family of demi-continuous operators such that all map bounded subsets of $[0, T] \times X$ to bounded subsets of $X$. Assume that for each $t \in[0, T], A(t)$ is accretive; then there is a unique solution to $(2.6)$ on $[0, T]$.

Proof. If we provide a local solution to (2.6) we can apply the argument of Theorem 1 to extend the solution to $[0, T]$. Our local existence argument follows Kato [7]. Let $\varepsilon_{n}+0$. Choosing $R, T_{0}, K>0$ as in Lemma 3.1 we define $u_{n}(t)$ for $t \in\left[0, T_{0}\right]$ by equation (3.2). We observe that

$$
d / d t\left(\left\|u_{n}(t)-u_{m}(t)\right\|^{2}\right)=-2\left(A(t) u_{n}\left(t-\varepsilon_{n}\right)-A(t) u_{m}\left(t-\varepsilon_{m}\right), F\left(u_{n}(t)-u_{m}(t)\right)\right\rangle
$$

where $F$ is the duality map. Using the accretiveness of $A(t)$ we obtain

$$
\begin{aligned}
& d / d t\left(\left\|u_{n}(t)-u_{m}(t)\right\|^{2}\right) \\
& \quad \leq-2\left(A(t) u_{n}\left(t-\varepsilon_{n}\right)-A(t) u_{m}\left(t-\varepsilon_{m}\right), F\left(u_{n}(t)-u_{m}(t)\right)-F\left(u_{n}\left(t-\varepsilon_{n}\right)-u_{m}\left(t-\varepsilon_{m}\right)\right)\right\rangle .
\end{aligned}
$$

Since $F$ is uniformly continuous the arguments of [7] and [8] are directly applicable to establish the uniform convergence of $u_{n}(t)$ to $u(t)$ on $\left[0, T_{0}\right]$. We apply the argument of Theorem 1 to see that $u(t)$ can be extended to a solution of (2.6) on $[0, T]$. The uniqueness of the solution follows from standard methods involving the accretiveness of $A(t)$.

\section{References}

[1] Fellx E. Browder, "Non-linear equations of evolution", Ann. of Math. (2) $80(1964), 485-523$.

[2] Shui-nee Chow and J.D. Schuur, "An existence theorem for ordinary differential equations in Banach spaces", Buzl. Amer. Math. Soc. 77 (1971), 1018-1020.

[3] J.B. Diaz and R.J. Weinacht, "On nonlinear differential equations in Hilbert spaces", Applicable Anal. 1 (1971), 31-41. 
[4] W.E. Fitzgibbon, "Weakly continuous accretive operators", BuZz. Amer. Math. Soc. 79 (1973), 473-474.

[5] W.E. Fitzgibbon, "Weakly continuous accretive operators in reflexive Banach spaces", Proc. Amer. Math. Soc. (to appear).

[6] Jerome A. Goldstein, "Uniqueness for nonlinear Cauchy problems in Banach spaces", Applicable Anal. (to appear).

[7] Tosio Kato, "Nonlinear semigroups and evolution equations", J. Math. Soc. Japan 19 (1967), 508-520.

[8] Tosio Kato, "Accretive operators and nonlinear evolution equations in Banach spaces", Nonlinear functional analysis, 138-161 (Proc. Sympos. Pure Math. 18, Part 1, Chicago, Illinois, 1968. Amer. Math. Soc., Providence, Fhode Is land, 1970).

[9] R.H. Martin, Jr, "A global existence theorem for autonomous differential equations in a Banach space", Proc. Amer. Math. Soc. 26 (1970), 307-314.

[10] Robert H. Martin, Jr, "The logarithmic derivative and equations of evolution in a Banach space", J. Math. Soc. Japan 22 (1970), 411-429.

[11] L.A. Medeiros, "On nonlinear differential equations in Hilbert spaces", Amer. Math. Monthly 76 (1969), 1024-1027.

[12] G.A. Webb, "Continuous nonlinear perturbations of linear accretive operators in Banach spaces", J. Functional Analysis 10 (1972), $191-203$.

Department of Mathematics, University of Houston, Houston, Texas, USA. 